\title{
Jacques PALARD, (introd.), Halvdan SKARD, (préf.), Des dieux dans la ville. Le dialogue interculturel et interreligieux au niveau local
}

Strasbourg, Éditions du Conseil de l'Europe, 2007, 244 p.

Bérengère Massignon

\section{QpenEdition}

\section{Journals}

Édition électronique

URL : http://journals.openedition.org/assr/21633

DOI : 10.4000/assr.21633

ISSN : $1777-5825$

Éditeur

Éditions de l'EHESS

Édition imprimée

Date de publication : 31 décembre 2009

Pagination : $75-342$

ISBN : 978-2-7132-2218-4

ISSN : 0335-5985

\section{Référence électronique}

Bérengère Massignon, « Jacques palaRd, (introd.), Halvdan SKARD, (préf.), Des dieux dans la ville. Le dialogue interculturel et interreligieux au niveau local », Archives de sciences sociales des religions [En ligne], 148 | octobre-décembre 2009, document 148-100, mis en ligne le 27 janvier 2010, consulté le 21 septembre 2020. URL : http://journals.openedition.org/assr/21633 ; DOI : https://doi.org/10.4000/ assr. 21633

Ce document a été généré automatiquement le 21 septembre 2020.

(C) Archives de sciences sociales des religions 


\section{Jacques PALARD, (introd.), Halvdan SKARD, (préf.), Des dieux dans la ville. Le dialogue interculturel et interreligieux au niveau local}

Strasbourg, Éditions du Conseil de l'Europe, 2007, 244 p.

\section{Bérengère Massignon}

\section{RÉFÉRENCE}

Jacques PALARD, (introd.), Halvdan SKARD, (préf.), Des dieux dans la ville. Le dialogue interculturel et interreligieux au niveau local, Strasbourg, Éditions du Conseil de l'Europe, 2007, 244 p.

1 Cet ouvrage collectif est issu d'un séminaire organisé par le Congrès des pouvoirs locaux et régionaux du Conseil de l'Europe, à Montchanin (France), en novembre 2006. Cette rencontre, alliant la présence d'élus et d'universitaires sur la question du dialogue interculturel et (surtout) interreligieux au plan local, s'est faite dans la ligne de la Déclaration du Conseil de l'Europe sur la diversité culturelle ou Déclaration de Faro (octobre 2005), soit pour le cinquantenaire du Conseil de l'Europe (CE), elle-même issue d'une recommandation du Conseil des Ministres du CE de décembre 2000.

2 Un double constat mène ce livre: l'accroissement du rôle des pouvoirs locaux du fait de la décentralisation et l'importance croissante des identifications religieuses individuelles et collectives, ce qui constitue une configuration inédite et peut-être une fenêtre d'opportunité. Pour Halvan Skard, Président du Congrès des pouvoirs locaux et régionaux du $\mathrm{CE}$, le dialogue tissé entre les pouvoirs locaux et des administrés de plus en plus divers sur le plan des origines et des convictions est «un mode d'expression des valeurs civiques, à commencer par celle d'égalité et d'équité; il est un puissant facteur de développement» (pp.10-11) et l'un des «meilleurs indicateurs tout à la fois de la 
démocratie territoriale, de la valeur de la coopération interrégionale et transfrontalière et de l'autonomie d'action des collectivités territoriales» (p.11). Autrement dit, il y aurait «une dimension citoyenne du religieux» (Cécile Jolly, Commissariat au Plan, France, citée p.31). L'enjeu du dialogue interreligieux est, dès lors, de tenir ensemble, sans irénisme ou naïveté, l'objectif de cohésion sociale et de reconnaissance de la pluralité (dialectique majorité/minorité; rapports anciennes et nouvelles minorités), au risque d'ethniciser les relations locales. Pour les politiques, le défi est de favoriser l'institutionnalisation des relations interreligieuses en évitant à la fois l'indifférence et l'ingérence. Ce dialogue a une forte portée symbolique, mais aussi une dimension fonctionnelle car il dispense «un esprit de mutualisation dont profite l'ensemble des champs d'intervention des politiques publiques locales: éducation, santé, développement durable, urbanisme, culture, sport, relations intergénérationnelles...» (p.11). Il est donc par nature «transversal» (p.243)

Du point des universitaires présents, les niveaux locaux, nationaux et internationaux s'entremêlent lorsqu'il s'agit de parler de dialogue interreligieux, même si le plan local constitue un niveau particulièrement intéressant d'expérimentation du fait de la proximité des acteurs religieux et politiques ainsi que de l'existence d'enjeux pratiques communs permettant une politique pragmatique, mais pas forcément moins passionnelle qu'au niveau national: qu'on songe aux mobilisations locales contre la construction de mosquées comme celle de Premià del Mar, à proximité de Barcelone, entre 2001 et 2002. Les monographies consacrées au cas espagnol (Flora Burchianti et Xabier Itçaina), comme au cas britannique (Anjum Anwar et Chris Chivers, deux acteurs $\mathrm{du}$ dialogue interreligieux dans le Lancashire; Brian Pearce) soulignent l'importance du choc des attentats du 11 septembre 2001 et des attentats de Madrid (mars 2004) ou de Londres (juillet 2005). Choc négatif qui diabolise «l'autre» musulman; mais choc aussi positif en ceci qu'il voit une mobilisation renouvelée des acteurs politiques nationaux et locaux en faveur d'un dialogue interreligieux susceptible de faire pièce à la prophétie de malheur d'Huntington d'un choc généralisé des civilisations, définies sur une base religieuse. L'article de Nikola Tietze, sur l'Allemagne, présente la voie originale hambourgeoise où un consensus a permis d'associer un acteur protestant en voie de sécularisation et des acteurs musulmans en quête de reconnaissance sur un projet commun: un enseignement de la religion pour tous, fait original dans un pays où la Constitution garantit l'enseignement confessionnel à l'école publique, dispositif ouvert aux catholiques, protestants et juifs, mais qui peine à faire une place à l'islam. Le décalage, que l'on observe aussi ailleurs en Europe, entre l'affaiblissement des religions historiques et le dynamisme de nouvelles minorités religieuses, débouche ici sur un compromis fécond. Cet article sur l'Allemagne a le mérite de replacer la politique religieuse des localités allemandes dans le temps long en rappelant que dans une première phase (années soixante) était pratiquée une "politique accommodante» «d'indifférence intéressée» en faveur des travailleurs migrants turcs: l'octroi du droit à une pratique religieuse, dont l'organisation était laissée aux pays d'origine, visait avant tout le retour au pays de ces travailleurs présentés comme temporaires. La dernière monographie porte sur la Russie postcommuniste. Agniezca Moniak-Azzopardi y décrit la «symphonie des pouvoirs» retrouvée entre l'État et l'orthodoxie, les conflits de celleci avec les catholiques (question uniate, création de quatre diocèses) et avec les protestants (enjeu du prosélytisme néo-protestant). L'auteure montre le relatif partage de souveraineté entre orthodoxie et islam selon les zones où ceux-ci sont majoritaires, sans oublier pour autant les tensions nées des deux guerres de Tchétchénie et la 
situation difficile des musulmans (mais aussi des bouddhiste, quatrième religion traditionnelle reconnue dans la Fédération de Russie) lorsqu'ils sont en situation de minorité face à une majorité orthodoxe, nationaliste et exclusive.

4 Outre ces cinq monographies, placées en seconde partie, comme autant d'exemples de «bonnes pratiques» (Espagne, Allemagne, Grande-Bretagne - deux articles très inégaux - et Russie), l'ouvrage problématise finement, dans sa première partie, les objectifs, les enjeux, la mise en œuvre et les conditions méthodologiques du dialogue interreligieux. Cette partie s'accompagne aussi de tableaux synoptiques sur la pratique différenciée des pays européens en matière de financement des cultes sur le plan national, de compétence en matière de culte des pouvoirs locaux, de religion dans l'enseignement..., ce qui fournit une utile base de données.

5 L'ouvrage se clôt sur la présentation de douze principes du dialogue interculturel et interreligieux pour les collectivités locales, évoqué en termes de "partenariat». Bref, cet ouvrage stimulant et riche d'études de cas est un parfait exemple de «rechercheaction» telle que l'encouragent les institutions européennes: praticiens comme universitaires y trouveront matière à réfléchir et à se documenter.

\section{AUTEURS}

BÉRENGÈRE MASSIGNON 